\title{
Design Challenges for Mobile and Wearable Systems to Support Learning on-the-move at Outdoor Cultural Heritage Sites
}

\author{
Alaa Alkhafaji 1,2,* 0000-0001-8625-886X, Sanaz Fallahkhair ${ }^{3}$ and Mihaela Cocea ${ }^{1}$ 0000- \\ 0002-5617-1779 \\ ${ }^{1}$ School of Computing, University of Portsmouth, Portsmouth, UK \\ \{Alaa.alkhafaji;Mihaela.cocea\}@ port.ac.uk \\ ${ }^{2}$ Department of Computer Science, College of Science, Mustansiriyah University, \\ Baghdad, IRQ \\ Alaa.alkhafaja@gmail.com
}

${ }^{3}$ School of Computing University of Brighton, Brighton, UK

S.fallahkhair@brighton.ac.uk

\begin{abstract}
This paper presents a novel set of design challenges for the development of mobile and wearable applications to be used at cultural heritage sites. These challenges were drawn out based on a user study that was carried out to evaluate a mobile application prototype, SmartC. SmartC was designed for supporting people in taking learning opportunities at sites whenever they need informally while they are on the move. Augmented reality and wearable computing, i.e. smart eye glasses, were used in this research with the aim of bringing the past to life, as well as enhancing visitors' engagement. SmartC was evaluated by 26 participants, potential end-users, in the field. The evaluation study mainly focused on the interaction and usability aspects, which contribute to the field of HCI. The paper outlines several issues and challenges that were identified based on the evaluation study, summarised as: (1) interaction design related; (2) wearable computing related; (3) surroundings and environment related; (4) learner related; (5) context of use; and (6) technical issues. This paper also identifies aspects that relate to methods to be used and applied to such cases for evaluation studies.
\end{abstract}

Keywords

Ubiquitous learning, mobile location-based services, outdoors cultural heritage, augmented reality, wearable computing

\section{Introduction}

Cultural heritage sites carry the historical values of society [1]. Cultural heritage, therefore, reflects the identity of societies [2] and it is considered the gateway people use to discover history. Thus, it is not surprising that researchers are constantly seeking a better way to enhance the interpretation of sites [3-6], which consequently enhance the 
experience and learning from them. Interpretation is not only about presenting factual information, but more importantly about evoking the emotional and intellectual connection between visitors and attractions [7], that would promote the sense of loyalty and belonging to the community, as well as increase awareness of cultural heritage places. To achieve this, the objective of sites' authorities [8] is to enhance sites' interpretation, as well as visitors' engagement, which will lead to increase attendance; this, in turn, would improve learning from sites. This is challenging nowadays due to few reasons, which include: (a) a lack of interest in visiting sites as often, which makes it challenging to derive people to visit sites [9]; (b) people do not see visiting sites as a learning trip but rather as entertainment; (c) the rapid pace of life does not leave much time for people to visit sites and explore them. As experiencing sites usually involves moving from one attraction to another and changing of contexts to acquire information, enabling learning while moving, which we refer to learning on-the-move, for this purpose it would be an excellent choice. Visitors, often visit sites with friends and family to socialise and at the same time experience the site and feel the place [10]. This might cause visitors to spend a limited amount of time to explore sites and learn about them, as they would prefer to stay with the group most of the time. Thus, it could be essential to have a tool that enables them to stay with the group most of the time, while simultaneously learning about the attractions [11]. Additionally, visitors often have a limited amount of time they are willing to spend at sites, as they need to return to other activities after the visit. Additionally, it would be more challenging if there are kids within a group of visitors, as they are usually not as patient as adults. Technology would be an excellent tool for this, which could be achieved by utilising location-based notification to deliver information regarding the history of and the available services at nearby sites. This has not been given a great attention in the literature as shown in the next section.

Although, technologies have been introduced to enhance visitors' experience, most of them are introduced with respect to indoors cultural heritage [12-14]. Emerging technologies, such as immersive and ubiquitous technologies have a great potential to enhance engagement at sites as well as visitors' experience [15]. They would bring the past closer and help visitors experience life back in time [16], but so far, they have been dedicated mainly for indoor settings, such as museums and galleries. However, outdoors cultural heritage is as important as indoors, and they might need extra attention, as usually attractions are distributed around cities with no members of staff available, but with labels and sometimes limited audio devices [17]. Additionally, the context of outdoors sites is different than indoors where variables such as weather and level of brightness (i.e. sun light), are easy to control. Based on the literature review in the next section, few studies were dedicated for outdoor settings. Also, augmented reality (AR) technology has been introduced to enhance interaction at cultural heritage sites but, again, mainly for indoor settings, as explained in the next section. However, using it in outdoor contexts has different challenges, which have not been explored greatly in the literature, as few studies have been conducted outdoors. Consequently, there is a need to explore further and deeper to better understand how variables of outdoor settings would affect the experience, and how visitors will deal with them; this would help researchers address challenges that might arise.

Based on the review outlined in the next section, there is a lack of technologies that help visitors of outdoor sits to learn-on-the-move and provide them with the right 
information at the right time based on location, automatically without any intervention from users. Such technology would be very helpful for visitors who are in a new place and do not know what points of interest may be nearby; it would also help visitors to smartly invest the time of the visit by receiving information while moving around individually or with a group. Furthermore, few studies introduced features to show how attractions appeared in the past using AR, which could be a very interesting feature for enhancing visitors' engagement according to previous studies [9, 17], that would also enhance the sites' interpretation, which is very important as culture heritage reflects the identity of society [18]. Consequently, it would enhance attendance, which is always the objective of cultural heritage authorities [8]. Therefore, in the long-term, that would have a great impact on conserving sites as the visitors' attendance is a key aspect that contributes to sites maintenance [8], which in turn contributes to a better economy. Another important aspect of showing attractions how they looked in the past, is that it brings the past to life, which would reinforce the link between the present and the past, which, in turn, would help stimulate the perpetuation of culture [19].

This paper presents a user-study in the field for the evaluation of a mobile app that adopts smart and ubiquitous learning technology, SmartC. SmartC was designed to provide a learning tool to be used at outdoor cultural heritage sites informally. The study provided an insight into how visitors react to using an AR app in outdoor settings of cultural heritage. The results of the study helped outline a set of design challenges for designing such services, which would assist researchers who work in this area to overcome challenges that might arise. SmartC was designed to assist visitors of cultural heritage take learning opportunities whenever they need regardless of time and place utilising mobile and wearable computing, i.e. smart eyeglasses. The smart eyeglasses would help free visitors' hands and enjoy the experience while at the same time acquiring information. Additionally, they would help them look at the actual artefact while acquiring information, which would support the association of the given information with the corresponding artefact [17]. SmartC was designed to help visitors learn informally whilst on-the-move by delivering instant information automatically based on location regarding cultural attractions and artefacts when passing by. Additionally, SmartC provides a service to show how attractions appeared back in time using AR technology. SmartC was developed based on a framework for developing such services that was introduced in previous research $[11,17]$, which was formulated based on three empirical studies to gather user requirements; the user study presented in this paper was conducted to evaluate SmartC in the field with 26 potential end-users. The focus of the evaluation study was mainly on the interaction between users and the app, as it is considered a key factor of user satisfaction that would significantly enhance their engagement [20,21]. Design challenges were identified during the study for designers to keep in mind when designing such services, which are outlined in this paper. Additionally, the limitations of the study setting are discussed, which would be helpful information in guiding researchers in setting such studies. A scenario-based design method was used to depict the context of use to guide the design. The adopted scenario was developed based on general requirements for designing such services that were identified in a previous study [22]. The next section provides an overview of similar technologies and highlights their limitations.

\section{Related work}


Ubiquitous computing offers technology that interweaves into our lives and the surrounding environment in an unobtrusive way [23, 24]. It offers features that allow people to be freed from the restriction of time and place, which would be an excellent choice for enhancing the experience at cultural heritage sites, as the experience at sites involves a lot of changing in contexts, and it happens at different times. It is contextaware computing which allows visitors to receive information based on the context while they are moving. This section provides an overview regarding similar technologies and highlights their limitations.

Mobile technology has been increasingly utilised to support people experiencing cultural heritage sites. Studies such as in [25-28] have introduced mobile guides particularly for enhancing the experience at cultural heritage sites. However, none of them was introduced to provide visitors with information regarding the surrounding attractions based on location automatically while moving. Geo-focussed services have recently drawn a great attention especially for activities at outdoor settings. These services use mobile devices and the Global Position System (GPS) for doing activities based on location. It is considered as a good tool for encouraging people to socialise while doing physical activities (e.g. geo-caching) [29] and were mainly introduced for outdoor settings such as in [30-36]. However, none of them was dedicated for enhancing visitors' experience at outdoor cultural heritage sites for learning, nor were they utilised to understand visitors'/learners' behaviour and attitude while using such services in outdoor settings, with the aim to identifying design challenges.

AR technology has been recently utilised in cultural heritage contexts to enhance visitors' experience. Some projects have been designed for indoor cultural heritage sites such as in [7, 37-41]. Some others were introduced for outdoor settings such as in [4245]. Nonetheless, they have not introduced notifications on-the-move based on location and at the same time show how attractions looked in the past, which would be an essential feature for enhancing visitors' engagements, as well as sites' interpretation. Utilising wearable computing such as smart eye glasses to learn from cultural heritage sites is yet immature in the literature; a few technologies were introduced in this respect such as [12, 46-49]. Although these technologies utilised wearable computing, but they were not aimed at supporting visitors' learning on-the-move, where they receive right information at the right time based on location and at the same time helping them experience life back in time.

Several mobile apps were designed for indoor cultural heritage settings to be used at museums. For example as in $[13,14,50,51]$. Nevertheless, these technologies were not introduced to meet the particular need of outdoor setting, as a few things need to be considered in outdoors such as: the weather, the noise, and the daytime, which do not matter in indoor

More research was carried out with respect to outdoors cultural heritage in terms of field studies to evaluate similar technologies, such as in $[6,52,53]$, but none of them explored how visitors react to challenges that might arise at outdoor setting, nor observed how they perform in different learning modes to experience sites (i.e. individual and group). Additionally, none of them outlined design challenges for designing such services, nor provide guidance for researchers in setting such studies, that would assist researchers when conducting similar studies.

Despite the growing literature of ubiquitous learning technologies, there is still a lack of systems that support visitors intelligently to learn informally based on location at 
outdoors cultural heritage while moving without the need of intervention from users. Learning on-the-move would enhance learners/visitors' experience at cultural heritage sites as it saves the time and effort visitors spend looking for information regarding sites. In addition, none of the aforementioned systems provides the right information at the right time in a form of notifications based on location when learners pass nearby sites or attractions. This could be a very helpful feature for learners who are in new places where they do not know what is interesting surrounding them, which help them invest their visit's time effectively. SmartC is designed to help visitors of outdoor sites take learning opportunities on-the-move regardless of time and place, which is presented in this paper. SmartC utilises AR to enable visitors experience life back in time by providing a feature to show how sites looked back in time. This feature would be a good choice in helping people feel heritage places and experience life back in time; that would help them understand the local history $[8,54]$. Additionally, it utilises smart eye glasses to free visitors' hands and let them enjoy the experience without the need of carrying a mobile device all the time, while at the same time acquiring information. This would enhance the interaction between visitors and artefacts. SmartC was evaluated in the field with potential end-users. Deeper insights were obtained regarding how visitors/learners deal with challenges at sites. Consequently, design challenges for developing such services were identified to assist designers of such services, as a considerable amount of time and effort would be saved if they are considered. Such challenges have not been given a great attention in the aforementioned studies. Furthermore, limitations related to study settings that were identified during the study are outlined, which would guide researchers in setting such studies. Next section presents the scenario-based method design.

\section{Scenario-based design}

A scenario-based design method was used to visualise the context of use in order to implement the prototype [22, 55], which was developed based on a subset of general requirements for designing such services. It is important to note, the general requirements were extracted from a framework for designing such services [17]. The adopted general requirements (GRs) are:

1. The service should maintain a content object

2. The service should support learning on the move

3. The service should support learners interact with context easily and efficiently

4. The service should consider the challenges that might arise in using mobile devices in outdoor settings.

One scenario depicted the context of using augmented reality via smart eyeglasses, which is illustrated below.

Scenario: Mary is the mum of 16-year-old Amy. Mary is very passionate about cultural heritage and wants to get her daughter to learn about her culture. Amy does not find visiting cultural heritage sites very interesting as she sees sites as ruined abandoned places like a dead habitation. Mary found a mobile app that has an interesting service that enables visitors to experience life back in time, which helps visitors to be closer to the past. The service could be delivered through the mobile phone's camera or smart eyeglasses. Mary thought that might help changes Amy's perspective about historical places. This service enables visitors to travel inside history and watch people's life back in time. She took Amy to a historical place that used to be a battlefield back at a particular period of time and encouraged her to use this service. Amy chose to use the smart eye 
glasses with a headset as she thought that would enable her to immerse in the atmosphere. The service displayed a real situation of the life back in time. Amy imagined herself walking down a street watching and listening to events surrounding her. Amy enjoyed the experience a lot, which gave her a real picture of how life back in time used to be and she understood stories behind events that happened back in time. That experience helped her appreciate history and made her feel proud about her culture. It helped change her opinion about visiting cultural heritage sites; Amy now sees that place differently, as the site is brought to life and not only a bunch of abandoned bricks.

The scenario was further analysed to pull out more detailed requirements (low-level requirements - LRs), which then led to develop features and services that better serve this context, which are illustrated in the Table 1.

These features and services were implemented in a working system with the aim of evaluating them with potential end-users to extract their perspective regarding using it in context. The next section presents the architecture and the main interfaces of SmartC.

Table 1. LRs with the adopted features and GRs they are belong to

\begin{tabular}{|c|c|c|}
\hline GRs & LRs & Features \\
\hline 2 & $\begin{array}{l}\text { Delivering instant information regarding } \\
\text { historical places when passing by }\end{array}$ & $\begin{array}{l}\text { Receiving notifications on-the- } \\
\text { move }\end{array}$ \\
\hline $1 \& 3$ & $\begin{array}{l}\text { Provide different information format to } \\
\text { deliver historical information }\end{array}$ & $\begin{array}{l}\text { Multi-mode information format (i.e. } \\
\text { text, audio, video \& images) }\end{array}$ \\
\hline 3 & $\begin{array}{l}\text { Adopt a feature that enables learners to } \\
\text { immerse themselves in the experience and } \\
\text { use their senses to experience the life back } \\
\text { in time }\end{array}$ & $\begin{array}{l}\text { Use augmented reality to show how } \\
\text { sites appeared in the past }\end{array}$ \\
\hline 3 & $\begin{array}{l}\text { Allow learners to use wearable and } \\
\text { immersive technologies at sites }\end{array}$ & Harnessing smart eye glasses \\
\hline 4 & Handling the potential errors & Error and process messages \\
\hline
\end{tabular}

\section{SmartC - architecture and interfaces}

A smart and ubiquitous learning application prototype was developed, which is called SmartC. The aforementioned scenario guided the design of SmartC, which helped in developing features and services; this section presents the design and implementation of SmartC. SmartC is a native android app, which was designed for smartphones and to be used in outdoor cultural heritage settings. A Sony XPERIA android device was used throughout the design and implementation stage. Sony android smart eyeglasses were used in this research, which helped investigate how learners react to them in the field. 
SmartC adopted context-aware technology with the use of location-based services (LBS) to provide historical information on-the-move, harnessing mobile and wearable technologies. The implemented version of SmartC was designed around a set of general requirements that were set out based on a framework for such services [17]. It is an informal learning environment which delivers contextual historical information to enhance learning from outdoor cultural heritage sites, also to support people to learn about sites while they are doing their daily routines. The contextual information is delivered through the mobile device's screen and smart eye glasses based on location in the form of notifications. The notification comes up when learners pass close to an attraction to draw their attention that there is an attraction nearby, which could interest them. Learners have an opportunity to access more information regarding that attraction when viewing the notification message. The smart eyeglasses help learners to engage their sight with the attractions and artefacts simultaneously with receiving information.

This app uses geo-fence technology, which is placing a virtual boundary around a geographical area. It works when a user enters or leaves the area, which is identified by latitude and longitude of the area [56]. For this app, a circle shape of a radius of $100 \mathrm{~m}$ was used to identify the geographical area of each involved attraction.

The mobile device gets triggered when a learner enters that virtual zone, which it is tracked using the GPS of the device. The device pushes a notification to alert the learner when he/she gets close to an attraction. Notifications are pushed via the app through the mobile-based interface and the glasses-based interface simultaneously when the mobile device gets triggered (see Figure 1). It provides multimode information format to deliver the information. In addition, it utilises AR to show how attractions appeared in the past (see Figure $2 \& 3$ ).

SmartC was evaluated in the field by potential end-users to capture users' perspective regarding using the app in the context. The evaluation study is presented in the next section.

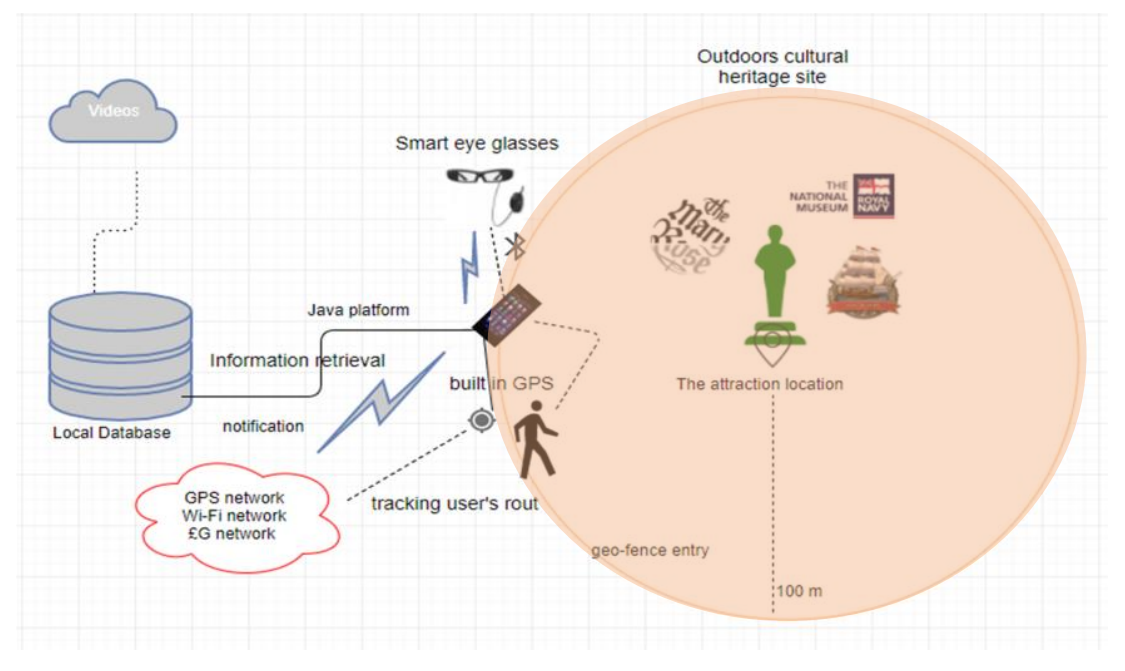

Fig. 1. Architecture of SmartC 

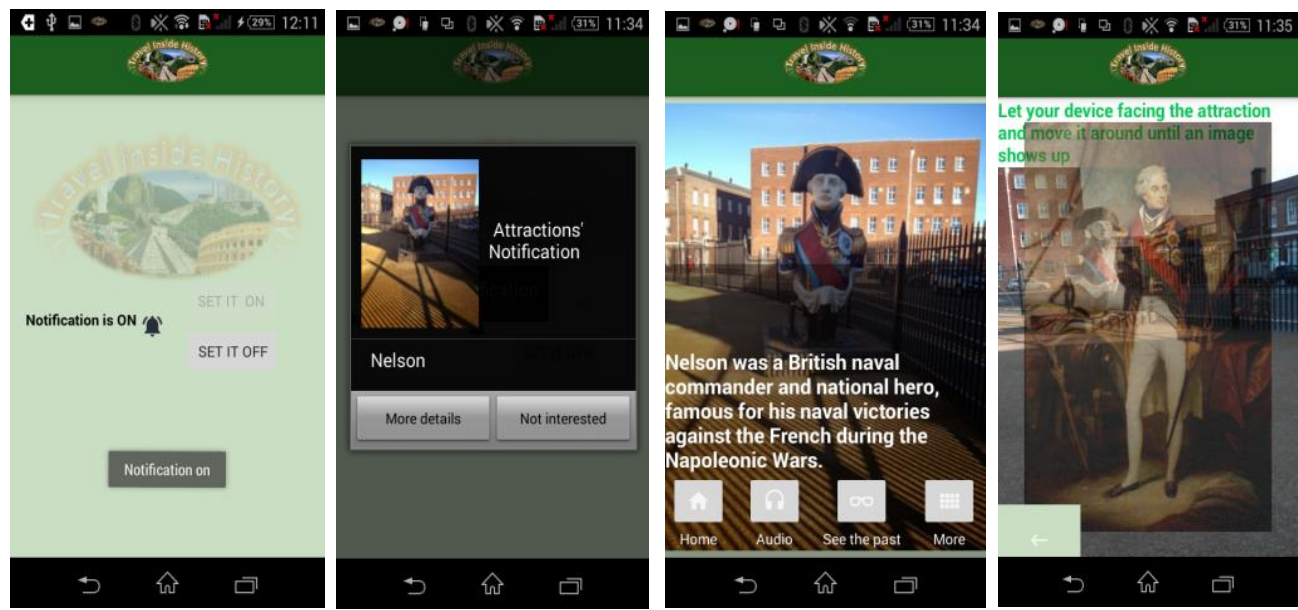

Fig. 2. Main screens of SmartC

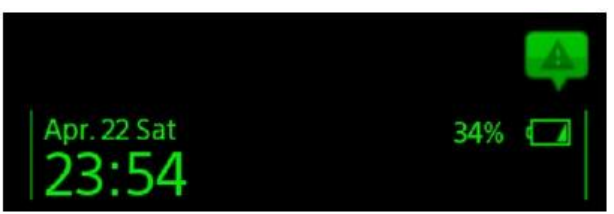

Fig. 3. Smart eye glasses interfaces

\section{The user study}

A user study was conducted to evaluate the SmartC app that was designed to be used at outdoor cultural heritage sites. This study was carried out to obtain users' feedback regarding their experience in using the app, which, in turn, helps to capture usability issues. Consequently, it helps setting out design challenges for designing similar technologies.

\subsection{Methods}

Three research techniques were combined in this study, which are: questionnaire, observation and a brief group interview. Emails and social media were used to target participants who were recruited using the convenience sampling method. Permission to use the Historic Dockyard in Portsmouth, UK, as a proof-of-concept was obtained from the authorities of the site. Participants were asked to use the app while walking around. The app was given to them in Android devices alongside a sheet to describe how it works. A simple statistical analysis using SPSS was used to obtain the mean for the data.

\subsection{Study Design}

Four sessions were carried out at the Historic Dockyard between $10^{\text {th }}$ and $12^{\text {th }}$ October 2016 to conduct this study; each session lasted around 2 hours; the tour and the discussion took around one hour each. This study used a combination of three techniques as it was mentioned: questionnaire, observation and group interview. The questionnaire technique 
involves different types of questions: scale of five, closed questions of two choices (yes/no) and open-ended questions. The questionnaire has three sections: usability evaluation, features rating, and overall acceptance.

Six categories, which were adopted from the ISO metric questionnaire, were involved in the usability section [57]. The categories include: suitability for learning, selfdescriptiveness, controllability, conformity with user expectations, error tolerance, and learnability [58]. The category "suitability of individualisation" was omitted, as the related features to this category were not included in this version of the app for pragmatic reasons such as personalising the app is required users to create their own account to personalise it based on their preferences. Additionally, to have a better experience of such features, users would be required to use it for a few days, which was not feasible in this evaluation study, where a few sessions of two hours were dedicated. Each included category involves a set of statements that participants were asked to state to what extent they agree or disagree with. A Likert scale of five was used, where 1=predominantly disagree and $5=$ predominantly agree.

This study also gathered users' feedback regarding the usefulness of features. Features include: (1) The audio explanations; (2) The attraction's image within the notification's dialogue; (3) Receiving notifications based on the location; (4) The text explanations; (5) The attraction's image within the attraction's page; (6) The attraction's image within the audio page; (7) The historical/ documentary videos; (8) Seeing how attractions looked in the past; (9) Take a photo; (10) Short messages giving feedback about tasks process; (11) Error messages; (12) Receiving notifications on the glasses.

Participants were asked to rate these features on a scale of five, where $1=$ useless and $5=$ useful. Furthermore, participants were asked regarding their overall attitude towards this app.

To obtain more in-depth opinions regarding the experience in using the app, a brief group interview was held with participants after filling the questionnaire. Participants were asked about their experience using the app and to point out any challenges that they experienced, if any. In addition, they were asked if they have any suggestions for improving the app. Notes were taken by the researcher to document participants' answers.
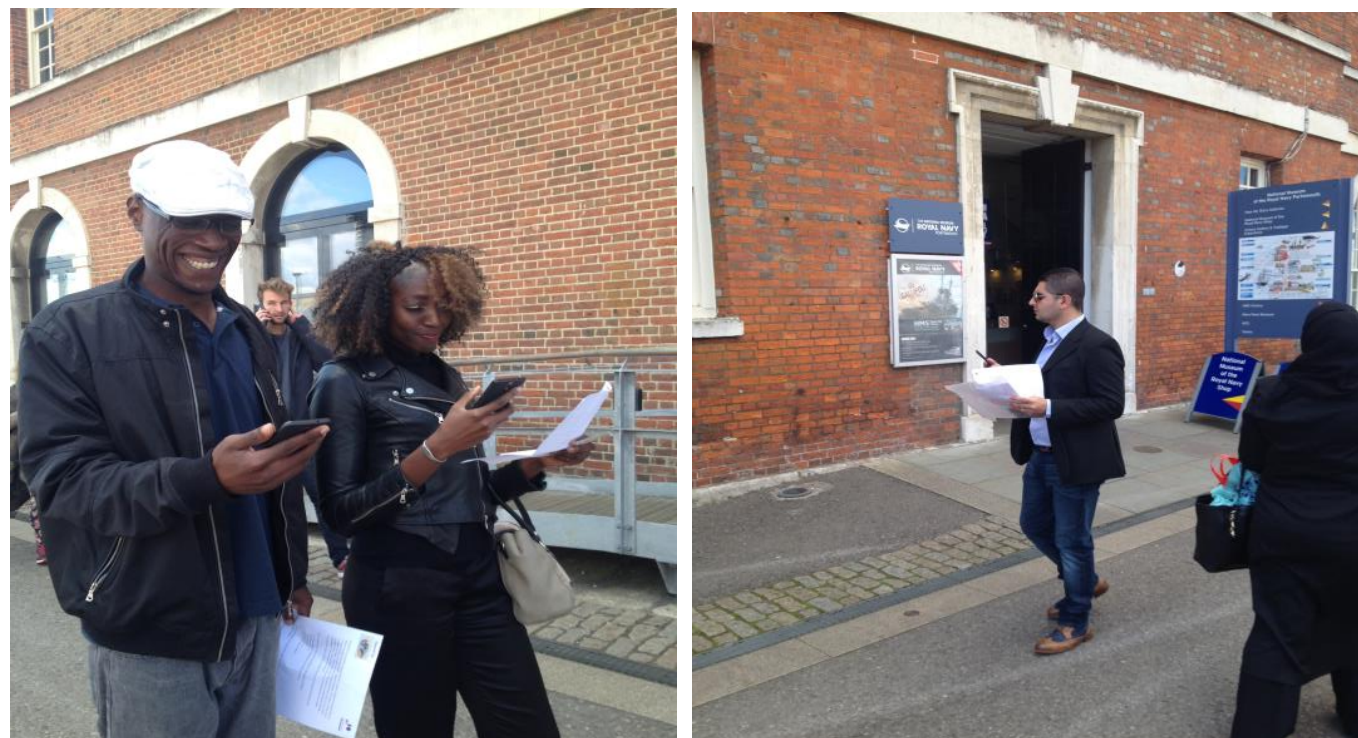

Fig.4. Participants while using the SmartC app in the field 
An observational technique was used to capture any problems or difficulties users might experience when using the app at outdoor settings. Although, filming was not allowed at the site due to the naval-base security issues, notes were taking during the tour.

\subsection{Participants}

26 participants took part in this study; all of them were residents in the UK/Portsmouth; their age ranged between 20 and 71. Participants were 8 females and 18 males from different nationalities: Iraq, Britain, Germany, Iran, Sweden, Libya, Nigeria, Senegal, Jordan and Colombia. Their occupations were: 19 students, one engineer, one project manager, one unemployed, one teaching fellow and three retired.

\subsection{Results}

The usability evaluation study with users was carried out to highlight the weak and strong points of the app from the users' perspective. Due to the nature of the informal learning as there is no standard scheme for the assessment of informal learning [59], it could be difficult to measure the effectiveness of learning and to assess how much information users take back from the visit. However, suitability for learning was assessed within the usability section. The main scope for this evaluation study was to assess the interaction design, which contributes to the field of mobile human-computer interaction (HCI). The results of the three techniques are given below.

The results of the usability questionnaire reveal positive attitudes from participants regarding the usability aspects of the app. The average of each category ranged between 3.06 and 4.25, which indicates participants found it usable and easy to use (see Table 2).

Table 2. The usability results

\begin{tabular}{|l|l|l|l|}
\hline \multicolumn{1}{|c|}{ The category } & The average & The category & The average \\
\hline Suitability for learning & 3.94 & Conformity with user expectations & 3.84 \\
\hline Self-descriptiveness & 4.05 & Error tolerance & 3.06 \\
\hline Controllability & 3.71 & Learnability & 4.25 \\
\hline
\end{tabular}
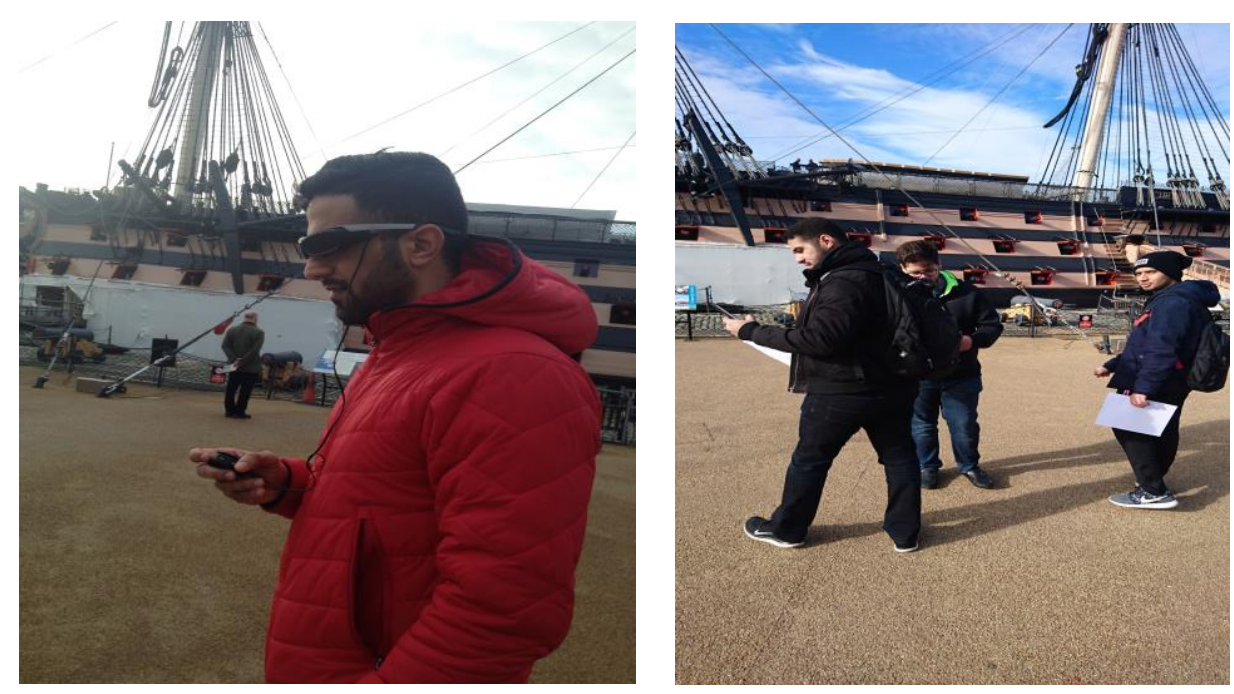

Fig.5. Participants while using SmartC via smart eye glasses and mobile phones in the field 
Alongside the evaluation of the interaction design, there was an assessment of how suitable the app was for learning. The results suggest the average of this category is 3.94 (see Table 2). This indicates SmartC is suitable for learning, which would facilitate acquiring information at outdoor cultural heritage sites effectively. In this light, although the evaluation from a learning perspective was relatively in a small scale, SmartC would be considered as a useful tool for learning.

Regarding the usefulness of the app, the results indicate that all features provided by the app are useful, as the mean ranges between 3.75 and 4.77. Participants liked receiving notifications based on location. Moreover, the results suggest that the audio explanation is the most popular information format amongst participants. Participants claimed that seeing attractions how they appeared in the past is very interesting and has a lot of potential. Three participants out of four reacted positively towards receiving notifications through the glasses and found it useful as it kept their hands free during the tour. One participant preferred to see the attractions with her own eyes instead of using the glasses; however, the device could be disabled when it is not needed. Participants highlighted some weaknesses in relation to the features of SmartC; some of these comments are given below:

"Lack of map, lack of direction, lack of [map] with direction of the attractions in historical time sequence"

"Hard to receive notification, simple design", "Volume of audios not high enough, little bit fiddly to see photo in past, not able to see the video after [leaving] attraction"

With respect to the overall acceptance, the vast majority of participants liked the app and stated they are happy to use it and recommend it to friends. Participants made some comments to illustrate their choice of why they would like to use the app. Some of these comments are given below:

"It provides flexibility of spreading knowledge, it is like you have one of those guidance in your pocket all the time."

"It is helpful, easier and lighter to use comparing to the old style ... guides for [sites]"

It is a good idea especially if you don't know the site"

"Idea of the app is quite interesting. It would be useful for open area like dockyard"

"Having an app for android on my phone is more feasible when visiting such sites rather than using devices provided by the sites, which need a bit of time for learning how to use it."

"Because of the content and seeing it in the past gives a good [idea] to how it was"

"It is very user friendly, you get interesting information that you would not get it just walking around, save spending on tourist' audio devices"

"I find it very useful and useful save a bit of time if you are in heritage"

One comment suggested to not use the app as one participant put: "No, because usually I walk around the attractions and read about the detailed information given and take my time to understand the writing". However, he stated that he would recommend it to friends.

The participants' perspectives were extracted post the tour during a brief group interview, which helped to obtain in-depth insight regarding their experience in using the app in the field. Participants were very positive towards it and found it interesting and 
easy to use. However, they pointed out some challenges that they experienced during the tour, which include:

1) keep receiving the same notification for the same attraction for a couple of times when passing nearby.

2) Losing the current notification (i.e. when viewing a notification for a certain attraction and then move to another one, could not go back to the previous one).

3) The old image in the "see it in the past" feature does not appear constantly as it is based on the location and it disappears once the device moves slightly.

4) An internet connection was needed to play the video which was not very good at the Historic Dockyard.

5) The audio did not stop when the participants used the back button of the device.

Several suggestions were given by participants to improve the app such as:

1) Add directions to the attractions.

2) Provide the distance to the attraction that users get notified about from their current location.

3) Make it easier to see what is nearby through a map with all attractions.

4) Provide alternatives to watch the video easily such as downloading.

5) Facilitate accessing the information of the attractions they passed by whenever they want.

6) Include public services in the notification service (e.g. cafes or toilets).

7) Take the daytime (day or night) into consideration when designing an app as the sun spells make it harder to see the screen sometimes.

8) Make the audio loud enough to be easily listened to within a group.

9) Provide a list of the nearby attractions instead of receiving notifications based on the location as one participant put. The perspective behind this suggestion was users need to get close enough to an attraction to get notified which may lead to missing an attraction if not. However, support people to learn on-the-move while they are doing their daily activities is the main point of providing notifications based on location.

The observational technique was used to observe how participants interact with the app during the tour and they deal with challenges that might arise. Fortunately, the weather was nice most days, with only one session on a cold day, which was not expected and consequently participants were not well clothed. That affected the tour slightly as they were not very comfortable walking around in outdoor settings. Participants divided themselves spontaneously into groups. Some participants walked around individually listening to an audio explanation and finding out more about attractions on their own. Some others used the app in a group using one device, and there were some participants who walked around as a group but using the app individually. All the groups were walking around, having conversations regarding the attractions, helping each other with using the app and discussing some weaknesses and strengths of the app.

Participants interacted with SmartC using mobile devices and smart eye glasses. Two narrative scenarios are given below to show how it was used. The scenarios are based on the real experiences that two participants had while using the app - the used names are not the participants' real names. In these scenarios, two examples of the involved attractions are used to depict the context.

(a) Scenario I: Jane used mobile phone only: 
Jane put the notification on and walked around with her mobile phone in her pocket. While walking, she sensed and heard a notification on her phone; she got the phone out of her pocket to find a notification regarding the lord Nelson's statue as shown in Figure 2. She had two options, which are: ignoring the notification or accessing more details. Jane chose to access more details as she knew a little about this famous figure in Portsmouth and was interested to know more; then she was taken to the corresponding attraction's page. She pressed on the "Audio" button and listened to the explanation while walking. When the audio has finished, Jane seemed amazed about the information she was given. So, she got excited to see how the lord looked back in time and what he used to wear; she pressed on the "See in the past" button and followed the instruction on the screen until she was able to see the old picture of the figure. Although it was not very easy to obtain this picture, she kept trying until it appeared, which indicates she has a great interest to experience life back in time. Then she moved to see the video, which she found interesting and added more information to what she listened to. This stimulated a short discussion between her and a group of participants who were close; the discussion was regarding: (i) events they watched in the video; (ii) the feature itself (i.e. the video format). Also, they mentioned the other features as well (details are given later in this section).

(b) Scenario II: Hani used mobile phone and smart eyeglasses:

Hani initiated the app on the phone to start the journey. He put his phone in his pocket and the glasses on. At the beginning, Hani could not see the glasses' interface as it needed to be in a specific position to be able to see the interface. Eventually, he managed to see the main interface, but the text was not very clear as the sunlight affected the visibility. Hani walked around with the smart glasses. While walking, he noticed a notification on the top right side of the glasses' interface as shown in Figure 3. Then he used the control pad to navigate through until he found the notification; however, Hani could not figure out how to use it easily. The notification was telling that the warship HMS Victory was close. He tapped on the control pad to access more details regarding the ship. The details were brief and just enough to draw his attention to the significance of the artefact, which he found interesting. He seemed satisfied as he was able to see the actual artifact through the glasses while reading the text on the glasses' screen. As he wanted to know more about it, Hani picked his mobile phone out to access more details (i.e. audio, video and see it in the past). Hani listened to the audio and used the "see it in the past" feature using his mobile phone as these are not available through the glasses in this version of SmartC. Nonetheless, he kept the glasses on while listening to the audio, which helped him associate the given information via the audio with the text on the glasses while looking at the ship. Whilst listening to the audio with the glasses on went smoothly, it seemed he had difficulty seeing the old picture of the ship; he needed take the glasses off to see the picture clearly as the transition between the two devices was not as smooth as he expected. He though the transition between the devices will be done automatically based on which device is active at that time, which was not the case. After Hani had enough information regarding the artefact, he had the glasses on again and placed his mobile phone back in his pocket; he enjoyed walking around and receiving notifications for the other attractions and artefacts via the glasses and only picked his mobile phone out when he needed to access more details.

The aforementioned scenarios show that SmartC is controlled mainly by the mobile phone. The role of smart eyeglasses in this version of SmartC is as a second screen to 
show visitors notifications and brief details regarding attractions and artifacts. This would help visitors have preliminary ideas regarding the attractions nearby, which would be enough to help them make a decision to access more details or not, before taking their mobile phone out. The two devices support each other to provide an easy and useful experience. The received notification is available in both devices simultaneously, but the glasses support the visitors' experience of sites without carrying their phones all the time; they do not need to take their mobile phone out their pocket if the received information is not very interesting for them. Hence, we could conclude that the devices support each other in terms of the usefulness and comfort of using the app, as the mobile phone provides more information (useful), and the glasses free visitors' hands while walking (comfort). However, this version of the glasses has a control pad that visitors needed to carry in their hands to control the glasses' interface. A newer version of smart glasses would free their hand completely.

It was noticed that participants were comfortable in using the app and navigating through and managed to use almost all the features easily. It is also true that participants who were walking in groups collaborated to find attractions and helped each other use the app and overcome challenges and interacted with their surroundings more often than those who walked alone. Interestingly, it was noticed that some participants walked away from the group when they discovered something interesting, to focus on the experience and find out more about a particular aspect of the attraction, and they re-join the group to have a conversation regarding what they found interesting. Additionally, participants showed more tolerance to error and challenges while with groups than those who walked individually. It was also noticed that participants who were always in a group, spent more time to finish the tour than those who experienced it individually. This may be due to the participants in the group setting enjoying socialising at the same time as experiencing the cultural heritage attractions; they took advantage of the 'take picture' feature to mark their experience, whereas those who were most of the time experiencing the site individually seemed to mostly enjoy being outdoors alongside feeling the place and constructing their own experience.

Most participants liked receiving notifications based on their current location; also, they liked the content especially in an audio format, which they found very useful. The service of "see it in the past" drew participants' attention and helped participants to engage with the attractions and to find out more information about them. Participants did not spend a considerable amount of time reading the texts on the screen, instead they played the audio to listen and walk. It was noticed that participants did not manage to find some attractions easily, for instance, they received a notification regarding HMS M.33 while they were near Nelson's statue, which they could not see, as HMS victory was blocking the sight. However, they emphasised that the provided picture on the app that illustrates how the attraction looks like helped them to find it. It was also noticed that the app did not work properly through some participants' own devices, which was because some required resources on the device were not enabled, such as camera and location-based services. In addition, several challenges were noticed during the tour:

1) $\mathrm{Wi}-\mathrm{Fi}, \mathrm{GPS}$ and $3 \mathrm{G}$ did not work properly which affected the performance of the app slightly and that was due to many radars around, as it is a naval-base site. Consequently, the notifications were sometimes hard to receive which needed to restart the notification (switch it off and switch it on again). 
2) Video did not play for a couple of times at some points, for the same previous reason, which needed participants to move their location slightly to be able to obtain a good signal to play the video.

3) The surrounding environment included challenges such as: daytime (day or night), weather (sunny or rainy), and noise. All these matters could also affect the users' experience, which are needed to be considered in designing such services.

4) Visitors' level of familiarity with technology could affect the experience. Some participants were less familiar in using mobile devices in general, which made the use of the app slightly harder.

5) The technical differences in operating systems of the android devices, as some devices show a good quality in picking locations more than the others. In addition, some explanation messages did not appear on some devices which made it challenging for some participants to figure out how some services work.

Overall, the results of both techniques, the interview and the observation, are consistent which gives a level of confirmation of the findings.

\section{Design Challenges - Discussion}

The user study presented in this paper helped in identifying issues and challenges in designing such services, which would help designers who are working in this field.

Participants, in general, found the app useful and easy to use and with a lot of potential for facilitating acquiring historical information on-the-move. In addition, the results indicate the app is suitable for learning. The results suggest that learners/visitors of cultural heritage sites enjoy the visit and the learning experience in groups as they can have a conversation regarding attractions and enjoy being outdoor with friends and family that clearly support social and collaborative learning. Additionally, the results show that participants were able to switch between different types of learning (individually and collaboratively) easily whenever they needed. They enjoyed being able to walk away from the group to further explore interesting aspects and return to the group to share the experience. This shows that technology would support both learning types and facilitate switching between them seamlessly.

Although the results suggest that SmartC is easy to use, some challenges and issues were identified in the study, which could be categorised into: (a) interaction design related, (b) wearable computing related, (c) surrounding environment related, (d) user related, and (e) technical issues related; details are given below.

\subsection{Interaction Design Related}

Some issues within the interaction design need to be considered to make the experience better using the mobile device.

a) The messages (error and feedback) need to be more obvious (e.g. keep it for longer, make it brighter, or make it in the middle of the screen);

b) The audio should stop when leaving the page using any means (the app standard button or the device standard button);

c) The volume of the audio and video need to be loud enough to be heard within a group; however, a headset splitter could be used to overcome this issue;

d) The augmented reality view (i.e. the image that illustrates how an attraction appeared in the past) in the "see it in the past" feature needs to be less faded and should be 
displayed for longer, even when changing the direction of the device slightly, to be easily seen.

\subsection{Wearable Computing Related}

Some issues were captured in using the wearable computing i.e. the smart eyeglasses, should be considered, which are limited in this version to:

a) The transition between the glasses and the mobile devices, which is needed to be done smoothly.

b) The usability aspect in terms of switching it off when it is not needed as it was challenging during the study.

c) The visibility of the text on the wearable devices' screen is challenging on a bright day, which needs to consider the adopted shades or colours.

d) The version and the brand of the utilised device (e.g. Sony smart glasses) needs to be chosen carefully to better serve the design of such services.

\subsection{Surrounding Environment Related}

The issues related to the surroundings include: daytime (day or night), weather (sunny, rainy or windy), the level of noise and the level of busyness of the heritage site. All these matters could also affect the experience, which could be addressed by providing different themes (i.e. colours) for day and night, and different modes for busy and quiet time.

\subsection{Learner Related}

User related issues include visitors' level of familiarity with technology and preferences, which could obstruct the experience. The results revealed that the level of familiarity with the mobile devices could be an issue in this context as it might make the use of the app challenging. In addition, users' preferences might cause them to not use the provided services if they do not meet their needs.

\subsection{Context of Use}

The results suggest that learners would like to use mobile devices to learn at sites in different context, which include:

(a) Individually and in-group: this study showed that participants like to learn in-group as it helped them to socialise and at the same time to share knowledge with others. In addition, they like being on their own as this helped in spending as much time as they wanted to explore sites. In short, learning in groups promotes a sense of community, but learning individually allows the learner to craft his/her own experience, both types of learning were seen in the evaluation study.

(b) On-site and off-site: results indicate visitors of sites enjoy walking around at sites and acquiring information in the context. Additionally, the results revealed that visitors of cultural heritage sites would like to have access to the acquired information not only in-site but off-site too, which would enable them to review the experience they had.

\subsection{Technical Issues}


Some technical issues came up during the study, as it was mentioned earlier, which include: (a) receiving the same notification more than once, (b) poor network signal which makes it hard to play a video or even receiving a notification, (c) android devices differences in terms of operating system, as some devices showed good quality in receiving notifications, and others showed poor quality in displaying some messages.

\section{Implications Related to Study Settings}

The setting of this empirical evaluation study involved several aspects that needed to be considered to make the contexts of use (the context in which the service will be evaluated) as ideal as possible in terms of setting the venue of the study.

In setting the venue, several aspects needed to be considered, such as: the weather as it is for outdoor settings; the size of the site in terms of the area and the number of attractions included, as it should have several attractions which are needed to be relatively close to each other to make it easier for participants to have the tour, but not too close that might affect the experience, i.e. receiving notifications. Additionally, in terms of the area, it needed to not be too large, just big enough for participants of all ages to have a proper tour at the site to visit all involved attractions comfortably in a short period of time. The Historic Dockyard was chosen, which has several attractions in outdoor settings that are relatively close to each other but not too close to affect receiving notifications. This choice also served the type of participation the evaluation studies adopted, which were organised groups and individuals.

Conducting a field study in the outdoor setting experienced some challenges such as the weather - as mentioned earlier, as there was a slight disruption due to unexpected weather conditions in one session, it was relatively cold, and participants were not prepared for such weather as it was not meant to be as cold in October in Portsmouth. However, the tour was enough to draw a conclusion regarding using the app. This limitation was addressed by taking advantage of cafes and seating areas with shelters to take refuge and discuss the experience amongst participants for a while.

Based on the discussion so far, choosing a site that is suitable for such empirical studies in the field is relatively subjective to a number of aspects such as: (a) the type of participants targeted, as if the targeted sample is elderly or children the criteria is different than adults who are willing to walk around a big site in a short period of time; (b) the duration dedicated for the study as dedicating two hours for a session is different than a day per session; (c) the type of participation, having an organised tour with a group of participants led by the researcher is different than individual session with the researcher, and this also is different than participants having a tour independently on their own based on their convenience. The first type is constrained by the time participants are willing to dedicate to the study as a whole, as they need to be all at the venue at a certain time and finish at a certain time; a group interview post-visit with all participants took part in each session to collect feedback. The second type is conducting individual sessions, which is slightly different from the first one, as it needs only to be convenient to one participant whilst the first one should be convenient to all participants taking part. In relation to the third one, where participation is not constrained to a number of hours but instead it is open to their time and preferences, this type is not necessarily to be in groups as often, and allows individuals to break from the group as needed; in this case, the venue could be in a spread area and the participants could choose a convenient time and which part of the tour they want to have based on their preferences. This type could 
be an excellent option for evaluating aspects such as personalisation as participants could create their own account and use the service for a few days to have the full experience to give useful feedback. The first type of participation was adopted in the study reported in this paper.

\section{Conclusions}

We have presented an evaluation study of SmartC, a mobile and wearable system for learning at outdoor cultural heritage sites. A scenario-based design method was used to depict the context of use to offer a useful insight for guiding the design of SmartC. SmartC was evaluated by end-users, in the field, which was conducted with 26 participants with the aim of capturing issues, and challenges that might occur when using a mobile application prototype at outdoor cultural heritage sites.

SmartC is a smart and ubiquitous learning prototype utilising mobile and wearable computing. It was designed to contribute to enhance the informal learning experience at outdoor cultural heritage sites. Smart eyeglasses were used to capture the users' perspective regarding using wearable computing, smart eyeglasses in particular. The results reveal that users liked the app and emphasised it was user-friendly and easy to use. In addition, the results indicate that there is a potential for using smart eyeglasses at sites as they are unobtrusive devices, which could be worn and taken off very easily.

Several issues were identified, which could be categorised as: (1) interaction design related; (2) wearable computing related; (3) surroundings and environment related; (4) learner related; (5) context of use; and (6) technical issues.

Several aspects were identified that need to be considered in setting such empirical studies, which would help researchers in setting a venue for such field studies.

There is an area that we envision to carry out further work, which is developing design recommendations for helping designers in designing such services.

\section{References}

1. Nuryanti, W., Heritage and postmodern tourism. Annals of tourism research, 1996. 23(2): p. 249-260.

2. González, M.V., Intangible heritage tourism and identity. Tourism management, 2008. 29(4): p. 807-810.

3. Angelaccio, M., et al. Smart and Mobile Access to Cultural Heritage Resources: a Case Study on Ancient Italian Renaissance Villas. in Enabling Technologies: Infrastructure for Collaborative Enterprises (WETICE), 2012 IEEE 21st International Workshop on. 2012. IEEE.

4. Bellotti, F., et al. Designing Cultural Heritage Contents for Serious Virtual Worlds. in Virtual Systems and Multimedia, 2009. VSMM'09. 15th International Conference on. 2009. IEEE.

5. Berndt, E. and J. Carlos, Cultural heritage in the mature era of computer graphics. Computer graphics and applications, IEEE, 2000. 20(1): p. 36-37.

6. McGookin, D., et al. Exploring Seasonality in Mobile Cultural Heritage. in Proceedings of the 2017 CHI Conference on Human Factors in Computing Systems. 2017. ACM.

7. Casella, G. and M. Coelho. Augmented heritage: situating augmented reality mobile apps in cultural heritage communication. in Proceedings of the 2013 
International Conference on Information Systems and Design of Communication. 2013. ACM.

8. Silberberg, T., Cultural tourism and business opportunities for museums and heritage sites. Tourism management, 1995. 16(5): p. 361-365.

9. Alkhafaji, A., et al. A survey study to gather requirements for designing a mobile service to enhance learning from cultural heritage. in European Conference on Technology Enhanced Learning. 2016. Springer.

10. Prentice, R., Experiential cultural tourism: Museums \& the marketing of the new romanticism of evoked authenticity. Museum Management and Curatorship, 2001. 19(1): p. 5-26.

11. Alkhafaji, A., S. Fallahkhair, and M. Cocea, Towards gathering initial requirements of developing a mobile service to support informal learning at cultural heritage sites. Cognition And Exploratory Learning In The Digital Age (CELDA 2015), 2015: p. 51.

12. Koren, I. and R. Klamma. Smart Ambient Learning with Physical Artifacts Using Wearable Technologies. in Intelligent Environments (Workshops). 2015.

13. Suriyakul, W. and G. Vavoula, Mobile Family Learning in the Science Museum, in mLearn17. 2017: Lamaca, Cyprus.

14. Vavoula, G., et al., Myartspace: Design and evaluation of support for learning with multimedia phones between classrooms and museums. Computers \& Education, 2009. 53(2): p. 286-299.

15. Weiser, M., The computer for the 21st century. Scientific american, 1991. 265(3): p. 94-104.

16. BBC. British Museum offers virtual reality tour of Bronze Age. 20154 August 2015; Available from: http://www.bbc.co.uk/news/technology-33772694.

17. Alkhafaji, A., The development of a theoretical framework for designing smart and ubiquitous learning environments for outdoor cultural heritage, in School of Computing. 2018, Portsmouth.

18. UNESCO, Managing Cultural Wold Heritage 2013, Paris: France: The United Nations Educational, Scientific and Cultural Organization (UNESCO).

19. Du Cros, H., A new model to assist in planning for sustainable cultural heritage tourism. International Journal of Tourism Research, 2001. 3(2): p. 165-170.

20. Soloway, E., M. Guzdial, and K.E. Hay, Learner-centered design: The challenge for HCI in the 21 st century. interactions, 1994. 1(2): p. 36-48.

21. Winters, N. and S. Price. Mobile HCI and the learning context: an exploration. in Proceedings of Context in Mobile HCI Workshop at MobileHCI05. 2005.

22. Carroll, J.M., Making use: scenario-based design of human-computer interactions. 2000: MIT press.

23. Dourish, P., What we talk about when we talk about context. Personal and Ubiquitous Computing, 2004. 8(1): p. 19-30.

24. Dingler, T., et al. uCanvas: A Web framework for spontaneous smartphone interaction with ubiquitous displays. in Human-Computer Interaction. 2015. Springer.

25. Candello, H., Multimedia information delivery on mobile cultural applications, in Universal Access in Human-Computer Interaction. Applications and Services for Quality of Life. 2013, Springer. p. 583-592. 
26. Suh, Y., C. Shin, and W. Woo, A mobile phone guide: spatial, personal, and social experience for cultural heritage. Consumer Electronics, IEEE Transactions on, 2009. 55(4): p. 2356-2364.

27. Van Aart, C., B. Wielinga, and W.R. Van Hage, Mobile cultural heritage guide: location-aware semantic search, in Knowledge Engineering and Management by the Masses. 2010, Springer. p. 257-271.

28. Chianese, A., F. Piccialli, and I. Valente, Smart environments and cultural heritage: a novel approach to create intelligent cultural spaces. Journal of Location Based Services, 2015. 9(3): p. 209-234.

29. Schlatter, B.E. and A.R. Hurd, Geocaching: 21st-century hide-and-seek. Journal of Physical Education, Recreation \& Dance, 2005. 76(7): p. 28-32.

30. Gray, H.R., Geo-caching: place-based discovery of virginia state parks and museums. Journal of Museum Education, 2007. 32(3): p. 285-291.

31. Tussyadiah, I.P. and F.J. Zach, The role of geo-based technology in place experiences. Annals of Tourism Research, 2012. 39(2): p. 780-800.

32. Pongpaichet, S., et al., Situation fencing: making geo-fencing personal and dynamic. 2013.

33. Clough, G., Geolearners: Location-based informal learning with mobile and social technologies. Learning Technologies, IEEE Transactions on, 2010. 3(1): p. 33-44.

34. Mendes, R.N., T. Rodrigues, and A. Rodrigues, Urban geo-caching. What happened in Lisbon during the last decade. International Archives of the Photogrammetry, Remote Sensing and Spatial Information Sciences, 2013: p. 2931.

35. Benford, S., et al., Can you see me now? ACM Transactions on Computer-Human Interaction (TOCHI), 2006. 13(1): p. 100-133.

36. O'Hara, K. Understanding geocaching practices and motivations. in Proceedings of the SIGCHI conference on human factors in computing systems. 2008. ACM.

37. Damala, A., et al. Bridging the gap between the digital and the physical: design and evaluation of a mobile augmented reality guide for the museum visit. in Proceedings of the 3rd international conference on Digital Interactive Media in Entertainment and Arts. 2008. ACM.

38. Fritz, F., A. Susperregui, and M. Linaza. Enhancing cultural tourism experiences with augmented reality technologies. in The 6th International Symposium on Virtual Reality, Archaeology and Cultural Heritage VAST. 2005.

39. Chang, Y.-L., et al., Apply an Augmented Reality in a Mobile Guidance to Increase Sense of Place for Heritage Places. Journal of Educational Technology \& Society, 2015. 18(2): p. 166-178.

40. Zoellner, M., et al. An augmented reality presentation system for remote cultural heritage sites. in Proceedings of the 10th International Symposium on Virtual Reality, Archaeology and Cultural Heritage VAST. 2009. Citeseer.

41. Haugstvedt, A.-C. and J. Krogstie. Mobile augmented reality for cultural heritage: A technology acceptance study. in Mixed and Augmented Reality (ISMAR), 2012 IEEE International Symposium on. 2012. IEEE.

42. Vlahakis, V., et al. Archeoguide: first results of an augmented reality, mobile computing system in cultural heritage sites. in Virtual Reality, Archeology, and Cultural Heritage. 2001. 
43. Liarokapis, F. and D. Mountain. A Mobile Framework for Tourist Guides. in Workshop on Virtual Museums, In Proc. 8th Int'l Symposium on Virtual Reality, Archaeology and Cultural Heritage (VAST'07), Eurographics, Brighton, UK. 2007.

44. Takacs, G., et al. Outdoors augmented reality on mobile phone using loxel-based visual feature organization. in Proceedings of the 1st ACM international conference on Multimedia information retrieval. 2008. ACM.

45. Caggianese, G., P. Neroni, and L. Gallo. Natural interaction and wearable augmented reality for the enjoyment of the cultural heritage in outdoor conditions. in International Conference on Augmented and Virtual Reality. 2014. Springer.

46. Feiner, S., et al., A touring machine: Prototyping 3D mobile augmented reality systems for exploring the urban environment. Personal Technologies, 1997. 1(4): p. 208-217.

47. Sparacino, F., The Museum Wearable: Real-Time Sensor-Driven Understanding of Visitors' Interests for Personalized Visually-Augmented Museum Experiences. 2002.

48. Leue, M.C., T. Jung, and D. tom Dieck, Google glass augmented reality: Generic learning outcomes for art galleries, in Information and Communication Technologies in Tourism 2015. 2015, Springer. p. 463-476.

49. Höllerer, T., et al., Exploring MARS: developing indoor and outdoor user interfaces to a mobile augmented reality system. Computers \& Graphics, 1999. 23(6): p. 779-785.

50. Hall, T. and L. Bannon, Designing ubiquitous computing to enhance children's learning in museums. Journal of Computer Assisted Learning, 2006. 22(4): p. 231-243.

51. Sung, Y.T., et al., Mobile guide system using problem-solving strategy for museum learning: a sequential learning behavioural pattern analysis. Journal of computer assisted learning, 2010. 26(2): p. 106-115.

52. Betsworth, L., et al., Performative technologies for heritage site regeneration. Personal and Ubiquitous Computing, 2014. 18(7): p. 1631-1650.

53. Andreoli, R., et al., A framework to design, develop, and evaluate immersive and collaborative serious games in cultural heritage. Journal on Computing and Cultural Heritage (JOCCH), 2017. 11(1): p. 4.

54. Poria, Y., R. Butler, and D. Airey, Links between tourists, heritage, and reasons for visiting heritage sites. Journal of Travel Research, 2004. 43(1): p. 19-28.

55. Carroll, J.M., et al., Requirements Development in Scenario-Based Design. IEEE Transactions On Software Engineering, 1998. 24(12).

56. Rodriguez Garzon, S. and B. Deva. Geofencing 2.0: taking location-based notifications to the next level. in Proceedings of the 2014 ACM International Joint Conference on Pervasive and Ubiquitous Computing. 2014. ACM.

57. Gediga, G., K.-C. Hamborg, and I. Düntsch, The IsoMetrics usability inventory: an operationalization of ISO 9241-10 supporting summative and formative evaluation of software systems. Behaviour \& Information Technology, 1999. 18(3): p. 151-164.

58. Fallahkhair, S., Development Of A Cross Platform Support System or Language Learners Via Interactive Television And Mobile Phone. 2009, Brighton. 
59. Skule, S., Learning conditions at work: a framework to understand and assess informal learning in the workplace. International journal of training and development, 2004. 8(1): p. 8-20. 